\title{
The use of elastic band exercise as a physical therapy intervention for improving shoulder function in older adults: a scoping review
}

\author{
Minjoon Kim ${ }^{1, *}$, Hironobu Kuruma ${ }^{2}$, Chirathip Thawisuk ${ }^{3}$ \\ 'Department of Rehabilitation, Tokyo General Hospital, Tokyo, Japan \\ 2Department of Physical Therapy, Tokyo Metropolitan University, Tokyo, Japan \\ ${ }^{3}$ Department of Occupational Therapy, Graduate School of Human Health Science, Tokyo Metropolitan University, Tokyo, Japan
}

Many older adults experience a decline in shoulder function due to aging. This decline leads to limitations in daily activities and a lower quality of life. The incorporation of physical therapy interventions through elastic band exercises has demonstrated improved overall physical faculties among older adults. However, there is limited literature regarding the effect of these interventions on shoulder function in older adults. This scoping review summarized the current literature regarding elastic band exercises targeting shoulder function in older adults. A systematic literature search was performed using the Scopus and PubMed databases. An additional manual search was conducted using the PEDro (Physiotherapy Evidence Database). Articles were included if they were published in a peer-reviewed journal in 2017-2021. After assessing eligibility, five randomized controlled trials articles were included in the analysis. We discovered that two types of elastic interventions were applied to older adults: namely, the TheraBand and tube bands. We observed heterogeneity in participant characteristics among the studies (healthy older adults, older adults with chronic obstructive pulmonary disease, and older adults with sarcopenic obesity). The duration of the exercise intervention ranged from 3 to 36 sessions. Only one study measured shoulder function as the primary outcome. Our findings suggest that elastic band exercises have been applied to older adults in various conditions and tended to be effective; however, evidence on this topic is insufficient.

Keywords: Shoulder, Resistance training, Geriatric, Exercise, Rehabilitation

\section{INTRODUCTION}

Aging is associated with a significant decline in the musculoskeletal system, which affects an individual's capacity to perform daily activities (Frontera, 2017). Many daily activities vital to independence, such as grooming, dressing, and household chores, require strength and coordination of the upper extremities (Lundgren-Lindquist and Sperling, 1983; Westerterp, 2000). Thus, a decline in the flexibility of the upper extremities due to the aging process contributes to decreased activity (Milanović et al., 2013). Among the upper extremities, the shoulder joint is the complex joint and it is essential for the function of the arms and hands, and dexterity (Bakhsh and Nicandri, 2018), which allows various functional abilities. However, increasing age is associated with decreased shoulder strength (Kinnucan et al., 2018) and the shoulder joint is the second most common joint site for pain among older adults. The prevalence of shoulder pain among the adult population ranges from $6.9 \%$ to $31 \%$. Furthermore, shoulder joint flexibility began to decline significantly after 70 years of age, at a rate of approximately $6^{\circ}$ per decade (Stathokostas et al., 2013), which may affect daily functioning. To treat and manage this condition, therapeutic exercise is commonly used within the practice of physical therapy.

A recent meta-analysis of exercise intervention for older adults showed the largest improvement for resistance training exercise among other types of interventions in terms of muscle strength
${ }^{*}$ Corresponding author: Minjoon Kim (D) https://orcid.org/0000-0003-1511-5604 Department of Rehabilitation, Tokyo General Hospital, 3 Chome-15-2 Egota, Nakano, Tokyo 165-8906, Japan

Email: ehrbs70@naver.com

Received: August 28, 2021 / Accepted: September 30, 2021
This is an Open Access article distributed under the terms of the Creative Commons Attribution Non-Commercial License (https://creativecommons.org/licenses/by-nc/4.0/) which permits unrestricted non-commercial use, distribution, and reproduction in any medium, provided the original work is properly cited. 
and general physical performance (Di Lorito et al., 2021). Resistance training exercises incorporating elastic devices have become increasingly interesting because they allow functional movement patterns and are more adaptable and accessible for individuals of various ages (Martins et al., 2015). Therapists can control exercise intensity by using different color codes or dimensions/forces of the elastic bands (Martins et al., 2015). Furthermore, elastic devices are portable and can be used almost anywhere and are low-cost and simple to maintain (Hostler et al., 2001). Regarding the effects of elastic resistance training, evidence from a recent systematic review showed that resistance exercise incorporating elastic bands was effective for improving flexibility, balance (Lopes et al., 2019), muscle strength, and functional capacity among older adults (de Oliveira et al., 2017).

However, little is known about the effects of this type of therapy on shoulder function in older adults. Since shoulder function plays an important role in the performance of daily activities and this function declines with age, it is necessary to summarize the current knowledge on this topic to guide clinical practices and identify gaps in the available literature. This scoping review summarized the current literature on elastic band exercises targeting shoulder function in older adults and identified potential avenues for future research.

\section{MATERIALS AND METHODS}

This scoping review was conducted following the article entitled "Guidance for conducting systematic scoping reviews" (Peters et al., 2015) and focused on the use of band exercises to improve shoulder function among older adults. This study defined elastic band exercise as resistance exercise involving the use of viscoelastic materials such as bands or tubes that exhibit nonlinear or viscous properties in combination with linear elastic properties.

\section{Search strategy}

The Scopus and MEDLINE (via PubMed) databases were searched during March 2021 using search terms including ("elder" OR "elderly" OR "older" OR "aged" OR "senior" OR "geriatric") AND ("resistance" OR "elastic" OR "rubber" OR "thera" OR "latex" OR "band" OR "program" OR "training" OR "exercise") AND ("shoulder" OR "shoulder joint" OR "shoulder). Manual searches were also performed in the PEDro (Physiotherapy Evidence Database) to ensure a comprehensive literature search. The eligibility criteria were set to identify articles relevant to the research question. The included articles were published in English between 2017 and 2021 within a peer-reviewed journal, described as a research study, and intended to determine the effect of band exercise on shoulder function in older adults (aged 65 years and above).

\section{Study selection}

After completion of the database searches, the citations and abstracts were compiled and entered into Covidence, where duplicate citations were removed. In the first stage, two reviewers independently screened the titles and abstracts. Conflicts were discussed until an agreement was reached between the reviewers. Full-text articles were obtained and then independently screened by the two reviewers and articles that met the inclusion criteria were included in the review. If the two reviewers could not reach an agreement on conflicting articles, a third expert reviewer (HK) was consulted. The details of the study selection process are presented in the Preferred Reporting Items for Systematic Reviews and MetaAnalyses (PRISMA) flow diagram (Fig. 1).

\section{RESULTS}

The analysis included five randomized controlled trials conducted in Taiwan, Korea, Canada, Brazil, and Iran (Table 1).

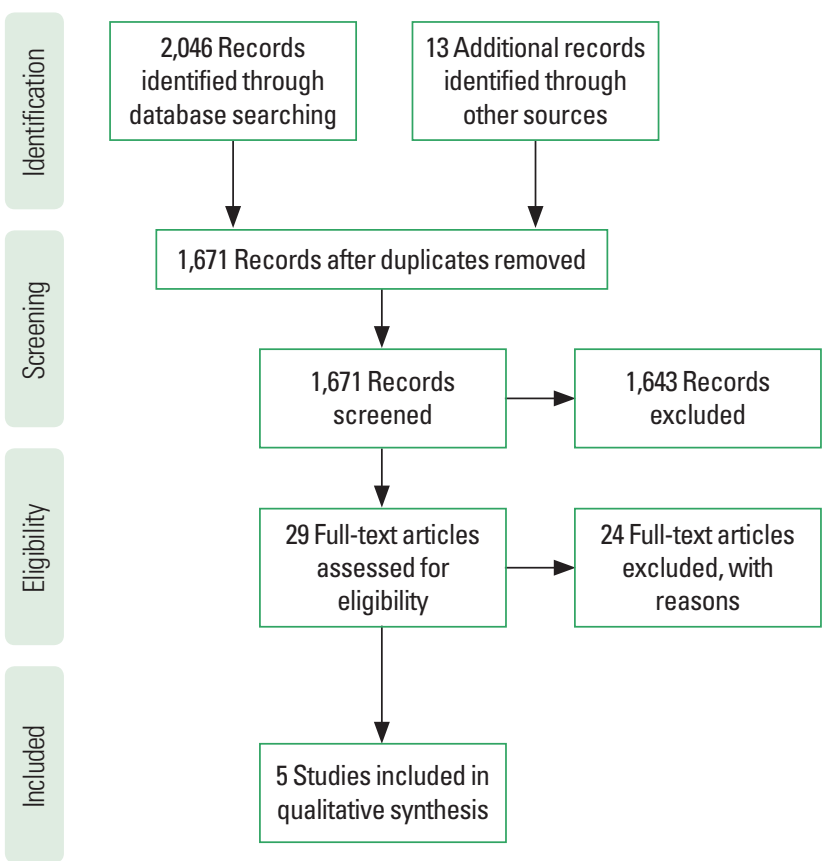

Fig. 1. Preferred Reporting Items for Systematic Reviews and Meta-Analyses (PRISMA) flow diagram of review process. 


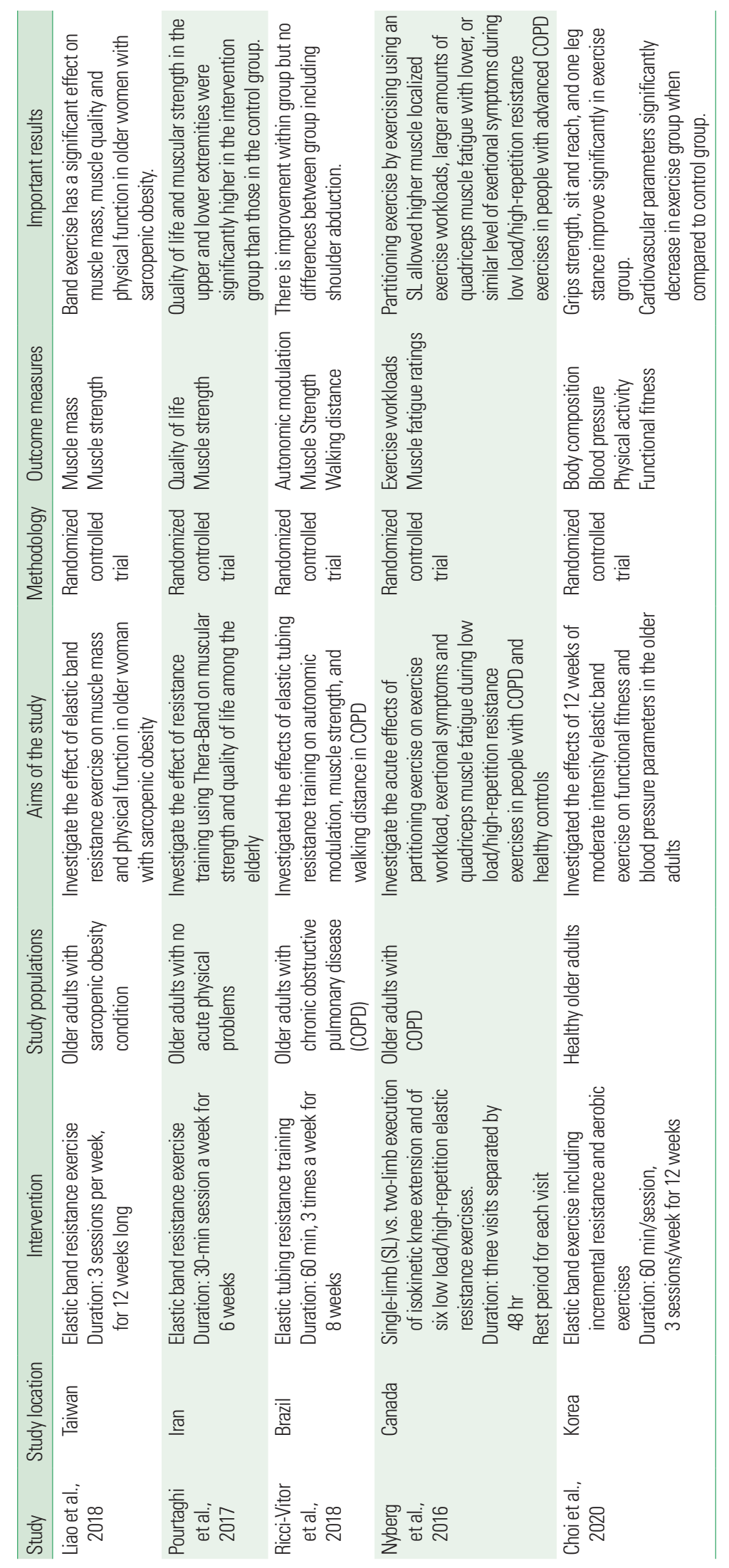




\section{Characteristic of the research subjects}

Among the included studies, the sample size ranged from 12 to 23 subjects, and all subjects were old people (65 years and older). However, the health conditions of the subjects varied. Two studies investigated the effects of band exercises in subjects with chronic obstructive pulmonary disease (COPD) compared to healthy subjects (Nyberg et al., 2016) and to COPD subjects who received another intervention (Ricci-Vitor et al., 2018). One study examined the effect of band exercises in older women with sarcopenic obesity (Liao et al., 2018). Another study was conducted in older adults in a community with no specific diagnosis, including healthy older adults and noncommunicable disease subjects (Pourtaghi et al., 2017). One study investigated the effects of band exercise in healthy old people, excluding those with medical problems (Choi et al., 2020).

\section{Band types}

Most of the included studies used TheraBands as the modality for resistance exercise (Liao et al., 2018; Nyberg et al., 2016; Pourtaghi et al., 2017). Two studies used elastic tubing as an exercise instrument, including Hyperform (Choi et al., 2020) and Lemgruber (Ricci-Vitor et al., 2018).

\section{Intervention}

Most of the included articles explained the details of band exercise intervention; for example, Liao et al. (2018) provided band exercises for three sessions a week, totaling 36 sessions, with each session comprising $10 \mathrm{~min}$ of warm-up, $40 \mathrm{~min}$ of band exercise, and $5 \mathrm{~min}$ of cool-down. The movements included seated chest press, seated row, seated shoulder press, knee extension, knee flexion, hip flexion, and hip extension. Similarly, the band exercises in the 36 sessions provided by Choi et al. (2020) consisted of front band raises, side lateral band raises, left and right shoulder waves, band hammer curls, band curls, triceps band extensions, triceps band kickbacks, band standing rows, band shoulder rotations, band squats, band deadlifts, standing band triceps extensions, standing band chest presser, upper bands, seated band rows, band leg raises, holding leg props, pelvis relaxations, and band crunches. However, in their study, the band exercises were performed for only $30 \mathrm{~min}$ for each session plus $15 \mathrm{~min}$ of warm-up and $15 \mathrm{~min}$ of cool-down. Pourtaghi et al. (2017) had a shorter duration, providing $30 \mathrm{~min}$ of band exercises per session, two sessions a week, for 6 weeks. Before each session, the subjects performed stretching exercises for $10 \mathrm{~min}$ and cooled down by jogging for $10 \mathrm{~min}$. The band exercises consisted of a chest press, shoulder press, front and back arm press, leg press, twin press, front thigh press, back thigh press, and posterior leg press. Ricci-Vitor et al. (2018) provided an intervention comprising 24 sessions, with three sessions per week. Each session lasted for $60 \mathrm{~min}$, including warm-up, cool-down, five movements and exercise, and a 2-min rest interval between each set due to the medical consideration of the subjects. However, their study did not provide details of the exercise movements. Nyberg et al. (2016) also provided band exercises for older adults patients with COPD. The exercises included latissimus rowing, leg curl, elbow flexion, chest press, shoulder flexion, and knee extension. However, they separated the band exercises into two types: single limb and two limbs. Because that study aimed to study the acute effects of band exercise, they provided only three sessions of band exercise.

\section{Outcome}

The primary outcomes of each study differed. Two studies using a band exercise intervention in older adults with COPD defined the outcome as measures of cardiopulmonary function such as heart rate variability (Ricci-Vitor et al., 2018) and pulmonary function (Nyberg et al., 2016). Muscle strength was measured as a secondary outcome in the included articles (Choi et al., 2020; Liao et al., 2018; Pourtaghi et al., 2017; Ricci-Vitor et al., 2018). The results showed the effectiveness of band exercises in improving muscle strength.

\section{DISCUSSION}

This scoping review aimed to summarize the current literature on elastic band exercises targeting shoulder function in older adults and to identify potential avenues for future research. Five articles met the inclusion criteria for this review. Our results demonstrated the lack of research focused specifically on the effect of elastic band exercise on shoulder function in older adults, with most of the studies including outcomes related to shoulder function as the secondary outcome. This was also observed in several systematic reviews that suggested the strong effect of elastic resistance band exercises on muscle strength (de Oliveira et al., 2017; Martins et al., 2013); however, few studies have described their effect separately on joints. In addition, the position statement of the National Strength and Conditioning Association pointed out that most resistance exercise protocols involve gross functional capacity for this reason and that single-joint protocols or outcomes are limited (Fragala et al., 2019).

Regarding the research participants, this scoping review found 
that older adults with various conditions received elastic band exercise therapy. However, a meta-analysis of elastic resistance exercise to increase muscle strength in older adults indicated that elastic resistance exercise was more effective in normal older adults and older adults with functional limitations. The effectiveness of this therapy was decreased in older adults with pathology (Martins et al., 2013). Therefore, when using elastic band exercise with older adults, therapists should consider the pathological conditions of individuals as the therapeutic effects might differ among individuals' conditions.

The resistance exercise interventions using elastic bands in this scoping review did not solely focus on shoulder joint movement or upper extremities as the lower extremities are also generally integrated into the exercise intervention. Regarding the duration of this intervention, we found that resistance exercise using elastic bands was usually performed for three sessions per week, ranging from 18 to 36 weeks. This is consistent with the recommendations of the National Strength and Conditioning Association of a resistance training program for older adults performed 2-3 times a week for each muscle group. This provides optimal stimulation to maximize the strength and size of the skeletal muscle in this population (Fragala et al., 2019).

Recently, the World Health Organization (WHO) declared 2020-2030 as the Decade of Healthy Aging, which aims to foster healthy aging and improve the lives of older people (World Health Organization, 2020). The WHO defines healthy aging as "the process of developing and maintaining the functional ability that enables wellbeing in older age" (Fallon and Karlawish, 2019; Michel and Sadana, 2017). In this context, the term "functional ability" includes the intrinsic capacity of the individual, environmental characteristics, and the interaction between these two components. Intrinsic capacity encompasses a person's mental and physical abilities, including their ability to move, think, see, hear, and remember. Several factors influence the level of intrinsic capacity, including the presence of diseases, injuries, and age-related changes (Beard et al., 2017).

Physical therapy is a healthcare profession with expertise in movement and exercise, which are essential skills to address the complexities of aging (World Confederation for Physical Therapy, 2019). Physical therapists can play not only remedial or rehabilitation roles for injuries but can also play a role in the prevention of injury, functional decline, and disability. Thus, physical therapists can help to promote healthy aging.

Our findings indicated that all the included articles demonstrated the effectiveness of elastic band exercises in promoting muscle strength in the shoulder complex. This is consistent with the results of a recent meta-analysis on exercise intervention in older adults, in which resistance training supported by nutritional supplementation significantly improved muscle strength. Thus, resistance training and multimodal exercises may improve general physical performance measures (Di Lorito et al., 2021). However, the findings of our review showed that the main focus of elastic band exercise is on increasing muscle strength among populations with reduced muscle strength or cardiopulmonary function.

To support the United Nations and WHO policies on healthy aging and the rapid increase in the number of older adults, physical therapists could consider the use of elastic band exercises as a preventive measure to maintain functional ability in older adults by managing intrinsic capacity such as muscle strength. Moreover, robust research on the use of elastic band exercises in terms of prevention needs to be highlighted.

This review was scoping by nature and not systematic due to the quality assessment of the included studies; thus, the results were not pooled. We observed heterogeneities in participants, type of elastic devices, elastic resistance exercise content, and outcome measures among these included studies. Despite these deficiencies, the initial overall findings were positive. The use of elastic band exercises appeared to be effective in improving shoulder function in older adults.

The scope of this review was limited to studies that applied elastic resistance exercises and measured the change in shoulder function in older adults. Thus, only five studies met the inclusion criteria. Additionally, the search terms used may not have been sufficiently sensitive, which was a potential limitation of this study.

Resistance exercise using elastic bands is gaining interest among physical therapy practitioners and has been applied to the older adult population. This scoping review summarized the current evidence on the use of elastic band exercises by physical therapists to improve shoulder function in older adults. The findings showed that while elastic band exercises have been applied to older adults with various conditions and appear to have positive effects, especially in muscle strength outcomes, there is insufficient evidence on shoulder function. Shoulder function is an important part of many functional activities and a large number of older adults face a natural decline in shoulder function. Thus, incorporating exercises to improve or maintain shoulder function has important applications. Furthermore, as elastic band exercises are generally effective at improving and maintaining musculoskeletal function in older adults, there is a considerable need to investigate how these exercises may affect shoulder function in this population. 


\section{CONFLICT OF INTEREST}

No potential conflict of interest relevant to this article was reported.

\section{ACKNOWLEDGMENTS}

The authors received no financial support for this article.

\section{REFERENCES}

Bakhsh W, Nicandri G. Anatomy and physical examination of the shoulder. Sports Med Arthrosc Rev 2018;26:e10-e22.

Beard JR, Araujo de Carvalho I, Sumi Y, Officer A, Thiyagarajan JA. Healthy ageing: moving forward. Bull World Health Organ 2017;95:730-730A.

Choi HM, Hurr C, Kim S. Effects of elastic band exercise on functional fitness and blood pressure response in the healthy elderly. Int J Environ Res Public Health 2020;17:7144.

de Oliveira PA, Blasczyk JC, Souza Junior G, Lagoa KF, Soares M, de Oliveira RJ, Filho PJBG, Carregaro RL, Martins WR. Effects of elastic resistance exercise on muscle strength and functional performance in healthy adults: a systematic review and meta-analysis. J Phys Act Health 2017;14:317-327.

Di Lorito C, Long A, Byrne A, Harwood RH, Gladman JRF, Schneider S, Logan P, Bosco A, van der Wardt V. Exercise interventions for older adults: a systematic review of meta-analyses. J Sport Health Sci 2021; 10:29-47.

Fallon CK, Karlawish J. Is the WHO definition of health aging well? frameworks for "health" after three score and ten. Am J Public Health 2019; 109:1104-1106.

Fragala MS, Cadore EL, Dorgo S, Izquierdo M, Kraemer WJ, Peterson MD, Ryan ED. Resistance training for older adults: position statement from the national strength and conditioning association. J Strength Cond Res 2019;33:2019-2052.

Frontera WR. Physiologic changes of the musculoskeletal system with aging: a brief review. Phys Med Rehabil Clin N Am 2017;28:705-711.

Hostler D, Crill MT, Hagerman FC, Staron RS. The effectiveness of 0.5-lb increments in progressive resistance exercise. J Strength Cond Res 2001;15:86-91

Kinnucan E, Molcjan MT, Wright DM, Switzer JA. A prospective look at the link between frailty and shoulder function in asymptomatic elderly individuals. Geriatr Orthop Surg Rehabil 2018;9:2151459318777583.

Liao CD, Tsauo JY, Huang SW, Ku JW, Hsiao DJ, Liou TH. Effects of elastic band exercise on lean mass and physical capacity in older women with sarcopenic obesity: a randomized controlled trial. Sci Rep 2018;8:2317.
Lopes JSS, Machado AF, Micheletti JK, de Almeida AC, Cavina AP, Pastre $\mathrm{CM}$. Effects of training with elastic resistance versus conventional resistance on muscular strength: a systematic review and meta-analysis. SAGE Open Med 2019;7:2050312119831116.

Lundgren-Lindquist B, Sperling L. Functional studies in 79-year-olds. II. Upper extremity function. Scand J Rehabil Med 1983;15:117-123.

Martins WR, de Oliveira RJ, Carvalho RS, de Oliveira Damasceno V, da Silva VZ, Silva MS. Elastic resistance training to increase muscle strength in elderly: a systematic review with meta-analysis. Arch Gerontol Geriatr 2013;57:8-15.

Martins WR, Safons MP, Bottaro M, Blasczyk JC, Diniz LR, Fonseca RM, Bonini-Rocha AC, de Oliveira RJ. Effects of short term elastic resistance training on muscle mass and strength in untrained older adults: a randomized clinical trial. BMC Geriatr 2015;15:99.

Michel JP, Sadana R. "Healthy aging" concepts and measures. J Am Med Dir Assoc 2017;18:460-464.

Milanović Z, Pantelić S, Trajković N, Sporiš G, Kostić R, James N. Age-related decrease in physical activity and functional fitness among elderly men and women. Clin Interv Aging 2013;8:549-556.

Nyberg A, Saey D, Martin M, Maltais F. Acute effects of low-load/highrepetition single-limb resistance training in COPD. Med Sci Sports Exerc 2016;48:2353-2361.

Peters MD, Godfrey CM, Khalil H, McInerney P, Parker D, Soares CB. Guidance for conducting systematic scoping reviews. Int J Evid Based Healthc 2015;13:141-146.

Pourtaghi F, Emami Moghadam Z, Ramezani M, Behnam Vashani H, Mohajer S. Effect of resistance training using thera-band on muscular strength and quality of life among the elderly. Evidence Based Care 2017;7:7-16.

Ricci-Vitor AL, Vanderlei LCM, Pastre CM, Ramos D, Ramos EMC, Ferreira Filho $\mathrm{C}$, Ferreira $\mathrm{C}$. Elastic tubing resistance training and autonomic modulation in subjects with chronic obstructive pulmonary disease. Biomed Res Int 2018;2018:9573630.

Stathokostas L, McDonald MW, Little RM, Paterson DH. Flexibility of older adults aged 55-86 years and the influence of physical activity. J Aging Res 2013;2013:743843.

Westerterp KR. Daily physical activity and ageing. Curr Opin Clin Nutr Metab Care 2000;3:485-488.

World Confederation for Physical Therapy. Description of physical therapy: policy statement [Internet]. World Physiotherapy; 2019 [2021 Aug 18]. Available from: https://world.physio/sites/default/files/2020-07/ PS-2019-Description-of-physical-therapy.pdf.

World Health Organization. Decade of healthy ageing plan of action [Internet]. Geneva (Switzerland): World Health Organization; 2020 [cited 2021 Aug 28]. Available from: https://www.who.int/initiatives/decadeof-healthy-ageing. 